\title{
Dairy Product Consumption and Risk of Non-Hodgkin Lymphoma: A Meta-Analysis
}

\author{
Jia Wang ${ }^{1}$, Xutong $\mathrm{Li}^{2}$ and Dongfeng Zhang ${ }^{1, *}$ \\ 1 Department of Epidemiology and Health Statistics, the Medical College of Qingdao University, \\ No. 38 Dengzhou Road, Qingdao 266021, China; wangjia900929@163.com \\ 2 Department of oncology, Second affiliated hospital, the Medical College of Qingdao University, \\ Qingdao 266071, China; lixutong1@sina.com \\ * Correspondence: zhangdongfeng@qdu.edu.cn; Tel.: +86-532-8299-1712; Fax: +86-532-8380-1449
}

Received: 26 November 2015; Accepted: 16 February 2016; Published: 27 February 2016

\begin{abstract}
Many epidemiologic studies have explored the association between dairy product consumption and the risk of non-Hodgkin lymphoma (NHL), but the results remain controversial. A literature search was performed in PubMed, Web of Science and Embase for relevant articles published up to October 2015. Pooled relative risks (RRs) with 95\% confidence intervals (CIs) were calculated with a random-effects model. The dose-response relationship was assessed by restricted cubic spline. A total of 16 articles were eligible for this meta-analysis. The pooled RRs ( $95 \%$ CIs) of NHL for the highest vs. lowest category of the consumption of total dairy product, milk, butter, cheese, ice cream and yogurt were $1.20(1.02,1.42), 1.41(1.08,1.84), 1.31(1.04,1.65)$, $1.14(0.96,1.34), 1.57(1.11,2.20)$ and $0.78(0.54,1.12)$, respectively. In subgroup analyses, the positive association between total dairy product consumption and the risk of NHL was found among case-control studies ( $R R=1.41,95 \%$ CI: $1.17-1.70)$ but not among cohort studies ( $R R=1.02$, 95\% CI: 0.88-1.17). The pooled RRs (95\% CIs) of NHL were $1.21(1.01,1.46)$ for milk consumption in studies conducted in North America, and $1.24(1.09,1.40)$ for cheese consumption in studies that adopted validated food frequency questionnaires. In further analysis of NHL subtypes, we found statistically significant associations between the consumption of total dairy product $(\mathrm{RR}=1.73,95 \% \mathrm{CI}: 1.22-2.45)$ and milk ( $\mathrm{RR}=1.49,95 \% \mathrm{CI}: 1.08-2.06)$ and the risk of diffuse large B-cell lymphoma. The dose-response analysis suggested that the risk of NHL increased by $5 \%$ (1.05 (1.00-1.10)) and 6\% (1.06 (0.99-1.13)) for each $200 \mathrm{~g} /$ day increment of total dairy product and milk consumption, respectively. This meta-analysis suggested that dairy product consumption, but not yogurt, may increase the risk of NHL. More prospective cohort studies that investigate specific types of dairy product consumption are needed to confirm this conclusion.
\end{abstract}

Keywords: dairy product; milk; non-Hodgkin lymphoma; meta-analysis

\section{Introduction}

Dairy product is an important part of diet in many countries around the world. Itcontains many essential nutrients, such as fats, proteins, minerals, vitamin $\mathrm{D}$, and other bioactive nutrients. Dairy product can increase the content of total body and lumbar spine bone mineral in children [1] and decrease the risk of cardiovascular disease [2], type 2 diabetes [3] and colorectal cancer [4]. However, in recent years, some studies have shown that excessive consumption of dairy product may be associated with several adverse health effects, for instance Parkinson's disease [5] and prostate cancer [6]. Therefore, it is necessary to further explore the health effect of dairy product on non-Hodgkin lymphoma (NHL). 
NHL refers to a heterogeneous group of malignant tumors of lymphoid tissue that differs from Hodgkin disease. Over the past few decades, the incidence and mortality of NHL has been increasing internationally [7-9]. It is estimated that there will be 71,850 new cases and 19,790 new deaths in the United States in 2015 [10]. In spite of the considerable public health significance, the etiology of NHL remains poorly understood. Recently, some studies have found that dietary factors may play a role in the development of NHL [11,12]. To date, several epidemiologic studies have explored the association between dairy product consumption and the risk of NHL. However, the results are inconsistent [13-28]. Therefore, we systematically conducted a meta-analysis to: (1) further explore the effect of total dairy product consumption on the risk of NHL; (2) further investigate the associations between specific types of dairy product consumption and the risk of NHL, including milk, butter, cheese, yogurt and ice cream; and (3) evaluate the possible dose-response relationships between the consumption of total dairy product and milk and the risk of NHL, respectively.

\section{Materials and Methods}

\subsection{Literature Search Strategy}

We conducted a literature search to identify relevant available articles published in English from PubMed, Web of Science and Embase up to October 2015. Search terms included "dairy" (or "milk" or "butter" or "cheese" or "yogurt" or "ice cream") and "non-Hodgkin lymphoma" (or "non-Hodgkin's lymphoma" or "NHL"). We also reviewed the reference lists of the included studies for undetected relevant studies.

\subsection{Inclusion Criteria}

The inclusion criteria are as follows: (1) case-control or cohort study published as an original study; (2) the exposure of interest were total dairy product, milk, butter, cheese, yogurt or ice cream; (3) the outcome of interest was non-Hodgkin lymphoma; (4) relative risk (RR) with $95 \%$ confidence interval (CI) (or data to calculate these) were provided; (5) the most recent and complete study was selected if data from the same population had been published more than once.

Two investigators (Jia Wang and Xutong Li) searched and reviewed all identified studies independently. If the two investigators disagreed about the eligibility of an article, it was resolved by consensus with a third reviewer (Dongfeng Zhang).

\subsection{Data Extraction}

The following information was extracted from each study by two investigators independently: first author's name, publication year, country in which the study was conducted, study design, follow-up duration, age range or mean age at baseline, sample size and number of cases, dietary assessment method, the type of dairy product, RR (we presented all results as RR for simplicity) with $95 \%$ CI for the highest versus lowest category of the consumption of total dairy product and specific types of dairy product, and variables adjusted for in each studies.

For dose-response analysis, the number of cases and participants (person-years), and RR (95\% CI) for each category of total dairy product and milk were extracted. The median or mean level of total dairy product and milk for each category was assigned to the corresponding RR for every study. If the upper boundary of the highest category was not provided, we supposed that the boundary had the same amplitude as the contiguous category. If intakes were reported in densities (i.e., g/1000 kcal), we estimated the absolute intakes by the mean energy intake of the participants [21]. When studies reported intakes in servings or times per day/week/month and did not provide a serving size, we converted them into grams per day by standard units of $244 \mathrm{~g}$ for milk and $177 \mathrm{~g}$ for total dairy product on the basis of serving sizes reported in the United States Department of Agriculture National Nutrient Database for Standard Reference [6,29]. We extracted RRs adjusted for the most confounders in the original studies. 


\subsection{Statistical Analysis}

Pooled measure was calculated as the inverse variance-weighted mean of the logarithm of RR with $95 \% \mathrm{CI}$ to assess the strength of associations between the consumption of total dairy product and specific types of dairy product and the risk of NHL, respectively. The DerSimonian and Laird random effect model (REM) was used to combine study-specific RRs (95\% CIs) [30]. The $I^{2}$ was adopted to assess the heterogeneity between studies $\left(I^{2}\right.$ values of $0 \%, 25 \%, 50 \%$ and $75 \%$ represent no, low, moderate and high heterogeneity, respectively) [31]. Meta-regression with restricted maximum likelihood estimation was performed to explore the potentially important covariates that might exert substantial impacts on between-study heterogeneity. $P$-values from meta-regression were calculated with a permutation test of 1000 to control the spurious findings [32]. Subgroup analyses were performed by study design, continent where the studies were conducted and dietary assessment method. Influence analysis was performed with one study removed at a time to assess whether the results could have been affected markedly by a single study [33]. Small-study effect was assessed with visual inspection of the funnel plot and Egger's test [34].

For dose-response analysis, a two-stage, random-effects, dose-response meta-analysis [35] was performed to compute the trend from the correlated log RR estimates across levels of total dairy product and milk, respectively. In the first stage, a restricted cubic spline model with three knots at the 10th, 50th, and 90th percentiles [36] of the levels of total dairy product and milk was estimated using generalized least-square regression, taking into account the correlation within each set of published RRs [37]. Then the study-specific estimates were combined using the restricted maximum likelihood method in a multivariate random-effects meta-analysis [38]. A $p$-value for nonlinearity was calculated by testing the null hypothesis that the coefficient of the second spline is equal to 0 .

All statistical analyses were performed with STATA version 12.0 (Stata Corporation, College Station, TX, United States). All reported probabilities ( $p$-values) were two-sided with $p<0.05$ considered statistically significant.

\section{Results}

\subsection{Literature Search and Study Characteristics}

We identified 199 articles by literature search, 173 of which were excluded after review of titles and abstracts (Figure 1). One additional article was found through the reference lists of included articles. Two articles with duplicate data from the same population, one article on the association between dairy product consumption and the risk of NHL mortality, one article on the association between "fruit and milk" dietary pattern and the risk of NHL, and seven articles without RR and/or 95\% CI were excluded. Finally, 16 published articles [13-28] were eligible for this meta-analysis.

In these included articles, seven studies were conducted in North America, two in Latin America, four in Europe and three in Asia. Thirteen articles adopted validated food frequency questionnaires (FFQs) to assess the dietary consumption, and others used FFQs. With regard to study design, 13 articles were case-control studies, and three were cohort studies. The detailed characteristics of the included studies are shown in Tables 1 and 2.

\subsection{Quantitative Synthesis}

The main results are summarized in Table 3.

\subsubsection{Total Dairy Product Consumption and the Risk of NHL}

Seven articles [13,15-17,21,22,28] with eight studies (five case-control studies and three cohort studies) were included, involving 4207 NHL cases. Among these studies, six were conducted in North America, one in Asia and one in Europe. All the studies adopted validated FFQs to assess total dairy product consumption. For the highest $v s$. lowest category of total dairy product consumption, the pooled RR of NHL was 1.20 (95\% CI 1.02-1.42, $I^{2}=42.7 \%, p$ heterogeneity $=0.094$, Figure 2). 
In subgroup analysis stratified by study design, the pooled RRs in case-control and cohort studies were $1.41\left(95 \%\right.$ CI $1.17-1.70, I^{2}=6.8 \%, p$ heterogeneity $\left.=0.368\right)$ and $1.02\left(95 \%\right.$ CI $0.88-1.17, I^{2}=0.0 \%$, $p_{\text {heterogeneity }}=0.988$ ), respectively (Figure 2 ). In subgroup analysis stratified by continent in which the studies were conducted, the association between total dairy product consumption and the risk of NHL was not statistically significant among studies conducted in North America. In the further analysis of NHL subtypes, we just found a statistically significant association between total dairy product consumption and the risk of diffuse large B-cell lymphoma (DLBCL) $(R R=1.73,95 \%$ CI 1.22-2.45, $I^{2}=0.0 \%, p_{\text {heterogeneity }}=0.670$ ).

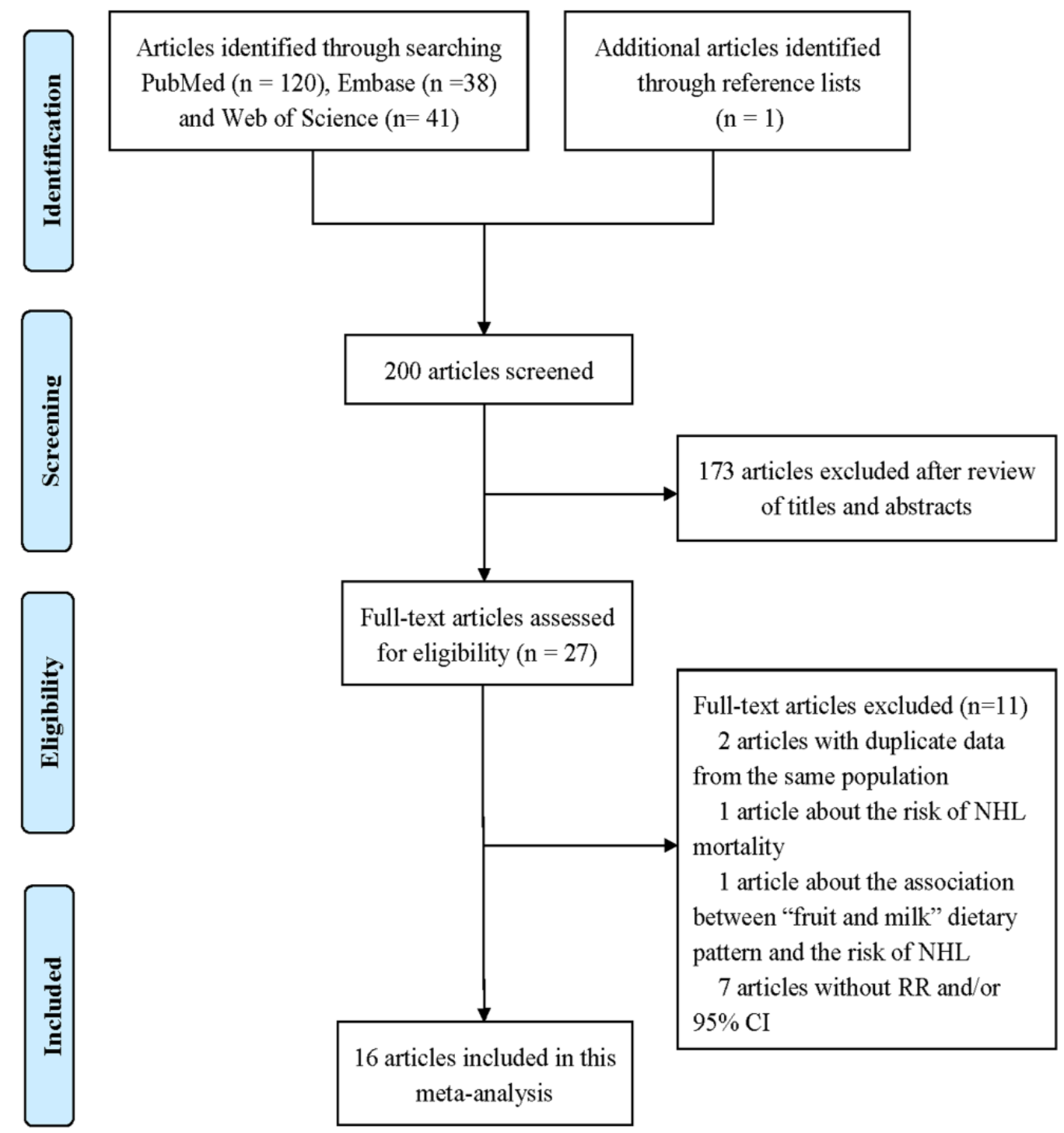

Figure 1. Flowchart of the selection of studies included in the meta-analysis.

For the dose-response analysis, data from five studies $[13,15-17,21]$ were used, including 1679 NHL cases. A linear relationship was found between total dairy product consumption and the risk of NHL ( $p$ nonlinearity $=0.91)$, and the RRs $(95 \%$ CIs) of NHL were $1.03(0.98-1.08), 1.10$ (0.95-1.27), 1.19 (1.00-1.41), 1.27 (1.06-1.51) and 1.42 (1.06-1.89) for 150, 390, 730, 1000 and $1500 \mathrm{~g} /$ day compared with $40 \mathrm{~g} /$ day, respectively. In addition, the dose-response analysis suggested that NHL risk increased by $5 \%(1.05(1.00-1.10))$ for each $200 \mathrm{~g} /$ day increment of total dairy product consumption (Figure 3). 
Table 1. Characteristics of case-control studies on dairy product consumption and the risk of NHL.

\begin{tabular}{|c|c|c|c|c|c|c|c|c|c|}
\hline Author & $\begin{array}{c}\text { Country } \\
\text { (Year) }\end{array}$ & Age & $\begin{array}{c}\text { Dietary } \\
\text { Assessment }\end{array}$ & $\begin{array}{l}\text { Participants } \\
\text { (Cases) }\end{array}$ & Gender & Exposure & Outcome & RR $(95 \% \mathrm{CI})$ & Adjustment for Covarianes \\
\hline $\begin{array}{l}\text { Balasubram-aniam, } \\
\text { G. [14] }\end{array}$ & India (2013) & $\begin{array}{l}\text { Cases } 46.1 \\
\text { Controls } 46.4 \\
\quad(\text { mean })\end{array}$ & FFQ & $1726(348)$ & M & Milk & NHL & $6.00(4.10,8.80)$ & $\begin{array}{l}\text { Cigarette smoking, bidi smoking, tobacco lime chewing, } \\
\text { the consumption of coffee, chicken, red-meat, eggs, fish, } \\
\text { chilly and vegetable, and exposure to pesticides and } \\
\text { cotton dust }\end{array}$ \\
\hline \multirow{3}{*}{ Zheng, T. [28] } & \multirow{3}{*}{$\begin{array}{c}\text { America } \\
(2004)\end{array}$} & \multirow{3}{*}{$21-84$} & \multirow{3}{*}{ Validated FFQ } & $1318(601)$ & \multirow{3}{*}{$\mathrm{W}$} & Total dairy & NHL & $1.80(1.20,2.80)$ & \multirow{3}{*}{$\begin{array}{l}\text { Age, BMI, family history of NHL in first-degree relatives, } \\
\text { and total energy intake }\end{array}$} \\
\hline & & & & $1305(594)$ & & Milk & NHL & $1.60(1.00,2.30)$ & \\
\hline & & & & $1205(494)$ & & Ice cream & NHL & $1.50(1.10,2.10)$ & \\
\hline \multirow{3}{*}{ Tavani, A. [26] } & \multirow{3}{*}{ Italy (1997) } & \multirow{3}{*}{$\begin{array}{l}\text { Cases } 58 \\
\text { Controls } 57 \\
\text { (median) }\end{array}$} & \multirow{3}{*}{ Validated FFQ } & \multirow{3}{*}{$1586(429)$} & \multirow{3}{*}{$\mathrm{M} \& \mathrm{~W}$} & Milk & NHL & $2.00(1.50,2.66)$ & \multirow{3}{*}{ None } \\
\hline & & & & & & Cheese & NHL & $1.37(1.03,1.82)$ & \\
\hline & & & & & & Butter & NHL & $1.78(1.21,2.62)$ & \\
\hline Ali, A. [13] & Oman (2013) & NA & Validated FFQ & $86(43)$ & $\mathrm{M} \& \mathrm{~W}$ & Total dairy & NHL & $0.81(0.29,2.23)$ & Age and sex \\
\hline \multirow{4}{*}{ Ward, M.H. [27] } & \multirow{4}{*}{$\begin{array}{l}\text { America } \\
(1994)\end{array}$} & \multirow{4}{*}{$\geqslant 21$} & \multirow{4}{*}{ FFQ } & \multirow{2}{*}{$714(171)$} & \multirow{2}{*}{$\mathrm{M}$} & Milk & NHL & $1.60(0.70,3.60)$ & \multirow{4}{*}{ Age } \\
\hline & & & & & & Cheese & NHL & $0.60(0.40,1.00)$ & \\
\hline & & & & \multirow{2}{*}{$676(144)$} & \multirow{2}{*}{$\mathrm{W}$} & Milk & NHL & $1.20(0.50,3.10)$ & \\
\hline & & & & & & Cheese & NHL & $0.70(0.40,1.30)$ & \\
\hline \multirow{6}{*}{ Chang, E.T. [15] } & \multirow{6}{*}{ Sweden (2005) } & \multirow{6}{*}{$\begin{array}{l}\text { Cases } 62 \\
\text { Controls } 59 \\
\text { (median) }\end{array}$} & \multirow{6}{*}{ Validated FFQ } & $1064(597)$ & \multirow{6}{*}{$\mathrm{M} \& \mathrm{~W}$} & & NHL & $1.50(1.10,2.20)$ & \multirow{6}{*}{ Age and sex } \\
\hline & & & & $595(128)$ & & Total dairy & DLBCL & $2.00(1.20,3.50)$ & \\
\hline & & & & $572(105)$ & & & FL & $1.20(0.60,2.20)$ & \\
\hline & & & & $614(147)$ & & & SLL/CLL & $1.50(0.90,2.60)$ & \\
\hline & & & & 1064 (597) & & Milk & NHL & $1.60(1.10,2.50)$ & \\
\hline & & & & $1064(597)$ & & Cheese & NHL & $1.40(1.00,2.00)$ & \\
\hline \multirow{2}{*}{ Purdue, M.P. [23] } & \multirow{2}{*}{ Canada (2004) } & \multirow{2}{*}{$20-74$} & \multirow{2}{*}{ Validated FFQ } & $5616(1631)$ & \multirow{2}{*}{$\mathrm{M} \& \mathrm{~W}$} & Milk & NHL & $1.12(0.95,1.31)$ & Age, sex, income adequacy, alcohol consumption, and \\
\hline & & & & $5554(1616)$ & & Cheese & NHL & $1.38(1.06,1.53)$ & \\
\hline Talamini, R. [25] & Italy (2006) & $\begin{array}{l}\text { Cases } 58 \\
\text { Controls } 63 \\
\text { (median) }\end{array}$ & Validated FFQ & $674(190)$ & $\mathrm{M} \& \mathrm{~W}$ & Milk Cheese & NHL NHL & $\begin{array}{l}0.91(0.54,1.54) \\
1.66(0.98,2.83)\end{array}$ & $\begin{array}{l}\text { Age, sex, center, education, place of birth, hepatitis } C \\
\text { virus test, and total energy intake }\end{array}$ \\
\hline De Stefani, E. [19] & $\begin{array}{l}\text { Uruguay } \\
(2013)\end{array}$ & NA & Validated FFQ & $3975(369)$ & $\mathrm{M} \& \mathrm{~W}$ & Milk & NHL & $2.98(2.23,3.98)$ & $\begin{array}{l}\text { Age, sex, residence, urban/rural status, education, BMI, } \\
\text { smoking intensity, total meat, alcohol drinking, mate } \\
\text { consumption, total meat, and total energy }\end{array}$ \\
\hline
\end{tabular}


Table 1. Cont.

\begin{tabular}{|c|c|c|c|c|c|c|c|c|c|}
\hline Author & $\begin{array}{l}\text { Country } \\
\text { (Year) }\end{array}$ & Age & $\begin{array}{c}\text { Dietary } \\
\text { Assessment }\end{array}$ & $\begin{array}{l}\text { Participants } \\
\text { (Cases) }\end{array}$ & Gender & Exposure & Outcome & RR $(95 \% \mathrm{CI})$ & Adjustment for Covarianes \\
\hline \multirow{18}{*}{$\begin{array}{c}\text { Ollberding, N.J. } \\
\text { [21] }\end{array}$} & \multirow{18}{*}{$\begin{array}{l}\text { America } \\
(2013)\end{array}$} & \multirow{18}{*}{$\begin{array}{l}\text { Cases } 58.6 \\
\text { Controls } 58 \\
\text { (mean) }\end{array}$} & \multirow{18}{*}{ Validated FFQ } & $793(333)$ & \multirow{18}{*}{$\mathrm{M} \& \mathrm{~W}$} & \multirow{4}{*}{ Total dairy } & NHL & $1.50(1.10,2.20)$ & \multirow{18}{*}{ Age, sex, educational attainment and total energy } \\
\hline & & & & $548(88)$ & & & DLBCL & $1.40(0.80,2.60)$ & \\
\hline & & & & $564(104)$ & & & FL & $1.50(0.90,2.60)$ & \\
\hline & & & & $485(25)$ & & & SLL/CLL & $3.00(0.90,9.50)$ & \\
\hline & & & & $793(333)$ & & \multirow{4}{*}{ Milk } & NHL & $1.60(1.10,2.30)$ & \\
\hline & & & & $548(88)$ & & & DLBCL & $1.80(1.00,3.10)$ & \\
\hline & & & & $564(104)$ & & & FL & $1.90(1.10,3.20)$ & \\
\hline & & & & $485(25)$ & & & SLL/CLL & $2.30(0.90,6.00)$ & \\
\hline & & & & $793(333)$ & & \multirow{4}{*}{ Cheese } & NHL & $0.90(0.70,1.30)$ & \\
\hline & & & & $548(88)$ & & & DLBCL & $0.90(0.50,1.60)$ & \\
\hline & & & & $564(104)$ & & & FL & $1.00(0.60,1.70)$ & \\
\hline & & & & $485(25)$ & & & SLL/CLL & $1.50(0.50,4.20)$ & \\
\hline & & & & $793(333)$ & & Ice cream & NHL & $1.40(1.00,2.00)$ & \\
\hline & & & & $793(333)$ & & \multirow{4}{*}{ Yogurt } & NHL & $0.80(0.50,1.20)$ & \\
\hline & & & & $548(88)$ & & & DLBCL & $0.60(0.30,1.20)$ & \\
\hline & & & & $564(104)$ & & & FL & $0.60(0.40,1.20)$ & \\
\hline & & & & $485(25)$ & & & SLL/CLL & $1.60(0.50,5.60)$ & \\
\hline & & & & $793(333)$ & & Butter & NHL & $1.00(0.70,1.40)$ & \\
\hline De Stefani, E. [18] & $\begin{array}{c}\text { Uruguay } \\
\text { (1998) }\end{array}$ & $20-84$ & FFQ & $\begin{array}{l}171(85) \\
152(75)\end{array}$ & $\begin{array}{l}\mathrm{M} \\
\mathrm{W}\end{array}$ & Milk & $\begin{array}{l}\text { NHL } \\
\text { NHL }\end{array}$ & $\begin{array}{l}1.03(0.47,2.28) \\
0.90(0.33,2.41)\end{array}$ & $\begin{array}{l}\text { Age, residence, urban/rural status, type of tobacco, beer } \\
\text { intake and "mate" /years Age, residence, urban/rural } \\
\text { status, year of diagnosis and parity }\end{array}$ \\
\hline
\end{tabular}


Table 1. Cont.

\begin{tabular}{|c|c|c|c|c|c|c|c|c|c|}
\hline Author & $\begin{array}{c}\text { Country } \\
\text { (Year) }\end{array}$ & Age & $\begin{array}{c}\text { Dietary } \\
\text { Assessment }\end{array}$ & $\begin{array}{l}\text { Participants } \\
\text { (Cases) }\end{array}$ & Gender & Exposure & Outcome & $\mathrm{RR}(95 \% \mathrm{CI})$ & Adjustment for Covarianes \\
\hline \multirow{18}{*}{$\begin{array}{c}\text { Charbonneau, B. } \\
\text { [16] }\end{array}$} & \multirow{18}{*}{$\begin{array}{l}\text { America } \\
(2013)\end{array}$} & \multirow{18}{*}{$\begin{array}{l}\text { Cases 60.9 } \\
\text { Controls 60.1 } \\
\quad(\text { mean) }\end{array}$} & \multirow{18}{*}{ Validated FFQ } & $1609(602)$ & \multirow{18}{*}{$\mathrm{M} \& \mathrm{~W}$} & \multirow{4}{*}{ Total dairy } & NHL & $1.12(0.79,1.60)$ & \multirow{18}{*}{ Age, sex, residence, and total energy } \\
\hline & & & & $1112(105)$ & & & DLBCL & $1.83(0.89,3.75)$ & \\
\hline & & & & $1153(146)$ & & & FL & $0.98(0.55,1.76)$ & \\
\hline & & & & $1225(218)$ & & & SLL/CLL & $0.88(0.52,1.47)$ & \\
\hline & & & & $1609(602)$ & & \multirow{4}{*}{ Milk } & NHL & $1.14(0.84,1.55)$ & \\
\hline & & & & $1112(105)$ & & & DLBCL & $1.85(1.01,3.40)$ & \\
\hline & & & & $1153(146)$ & & & FL & $0.99(0.58,1.70)$ & \\
\hline & & & & $1225(218)$ & & & SLL/CLL & $0.84(0.54,1.32)$ & \\
\hline & & & & $1609(602)$ & & \multirow{4}{*}{ Cheese } & NHL & $1.12(0.81,1.57)$ & \\
\hline & & & & $1112(105)$ & & & DLBCL & $1.06(0.52,2.12)$ & \\
\hline & & & & $1153(146)$ & & & FL & $0.94(0.53,1.69)$ & \\
\hline & & & & $1225(218)$ & & & SLL/CLL & $1.18(0.73,1.91)$ & \\
\hline & & & & 1609 (602) & & Ice cream & NHL & $2.45(1.80,3.34)$ & \\
\hline & & & & $1609(602)$ & & \multirow{4}{*}{ Yogurt } & NHL & $1.01(0.77,1.33)$ & \\
\hline & & & & $1112(105)$ & & & DLBCL & $0.88(0.49,1.57)$ & \\
\hline & & & & $1153(146)$ & & & FL & $1.12(0.70,1.81)$ & \\
\hline & & & & $1225(218)$ & & & SLL/CLL & $0.99(0.68,1.45)$ & \\
\hline & & & & 1609 (602) & & Butter & NHL & $1.29(0.99,1.69)$ & \\
\hline \multirow{5}{*}{ Mozaheb, Z. [20] } & \multirow{5}{*}{$\operatorname{Iran}(2012)$} & \multirow{5}{*}{$\begin{array}{l}\text { Cases } 51 \\
\text { Controls } 47 \\
\text { (mean) }\end{array}$} & \multirow{5}{*}{ Validated FFQ } & \multirow{5}{*}{$360(170)$} & \multirow{5}{*}{$\mathrm{M} \& W$} & Milk & NHL & $0.72(0.44,1.20)$ & \multirow{5}{*}{ None } \\
\hline & & & & & & Cheese & NHL & $1.38(0.79,2.40)$ & \\
\hline & & & & & & Ice cream & NHL & $1.05(0.65,1.71)$ & \\
\hline & & & & & & Yogurt & NHL & $0.32(0.18,0.55)$ & \\
\hline & & & & & & Butter & NHL & $1.34(0.76,2.37)$ & \\
\hline
\end{tabular}

Abbreviations: RR, relative risk; CI, confidence interval; BMI, body mass index; FFQ, food frequency questionnaire; M, men; W, women; NHL, non-Hodgkin lymphoma; DLBCL, diffuse large B-cell lymphoma; FL, follicular lymphoma; SLL/CLL, small lymphocytic lymphoma/chronic lymphocytic leukemia; NA, not available. 
Table 2. Characteristics of cohort studies on dairy product consumption and the risk of NHL.

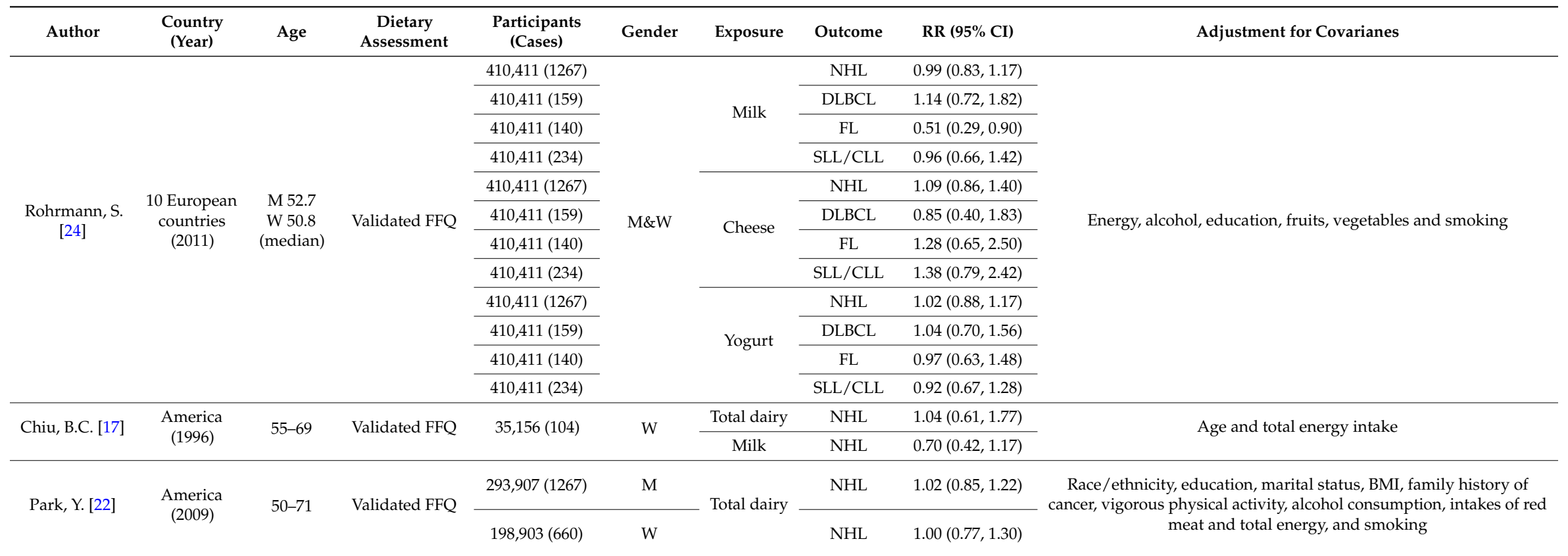

Abbreviations: RR, relative risk; CI, confidence interval; BMI, body mass index; FFQ, food frequency questionnaire; M, men; W, women; NHL, non-Hodgkin lymphoma; DLBCL,

diffuse large B-cell lymphoma; FL, follicular lymphoma; SLL/CLL, small lymphocytic lymphoma/chronic lymphocytic leukemia.3.2. Quantitative Synthesis 
Table 3. Summary risk estimates of the association between dairy product consumption and the risk of NHL and NHL subtypes.

\begin{tabular}{|c|c|c|c|c|c|c|c|}
\hline Exposure & Outcome & Subgroup & No. of Studies & No. of Cases & Pooled RR (95\% CI) & $I^{2}(\%)$ & $p_{\text {heterogeneity }}$ \\
\hline \multicolumn{8}{|c|}{ Total dairy product } \\
\hline \multirow{11}{*}{$\begin{array}{l}\text { Total dairy } \\
\text { product }\end{array}$} & \multirow{8}{*}{ NHL } & All studies & 8 & 4207 & $1.20(1.02,1.42)$ & 42.7 & 0.094 \\
\hline & & \multicolumn{6}{|c|}{ Study design } \\
\hline & & Case-control & 5 & 2176 & $1.41(1.17,1.70)$ & 6.8 & 0.368 \\
\hline & & Cohort & 3 & 2031 & $1.02(0.88,1.17)$ & 0.0 & 0.988 \\
\hline & & \multicolumn{6}{|c|}{ Continent } \\
\hline & & North America & 6 & 3567 & $1.17(0.98,1.40)$ & 46.6 & 0.095 \\
\hline & & Europe & 1 & 597 & $1.50(1.06,2.12)$ & NA & NA \\
\hline & & Asia & 1 & 43 & $0.81(0.29,2.25)$ & NA & NA \\
\hline & DLBCL & All studies & 3 & 321 & $1.73(1.22,2.45)$ & 0.0 & 0.670 \\
\hline & FL & All studies & 3 & 355 & $1.23(0.88,1.72)$ & 0.0 & 0.569 \\
\hline & SLL/CLL & All studies & 3 & 390 & $1.35(0.77,2.39)$ & 53.8 & 0.115 \\
\hline & & & Specific typ & f dairy product & & & \\
\hline \multirow{15}{*}{ Milk } & \multirow{12}{*}{ NHL } & All studies & 16 & 7109 & $1.41(1.08,1.84)$ & 88.6 & 0.000 \\
\hline & & \multicolumn{6}{|c|}{ Study design } \\
\hline & & Case-control & 14 & 5738 & $1.53(1.13,2.06)$ & 87.7 & 0.000 \\
\hline & & Cohort & 2 & 1371 & $0.91(0.68,1.22)$ & 36.8 & 0.209 \\
\hline & & \multicolumn{6}{|c|}{ Continent } \\
\hline & & North America & 7 & 3579 & $1.21(1.01,1.46)$ & 37.8 & 0.140 \\
\hline & & Latin America & 3 & 529 & $1.53(0.63,3.70)$ & 80.5 & 0.006 \\
\hline & & Europe & 4 & 2483 & $1.32(0.87,1.98)$ & 85.0 & 0.000 \\
\hline & & Asia & 2 & 518 & $2.09(0.26,16.71)$ & 97.7 & 0.000 \\
\hline & & \multicolumn{6}{|c|}{ Dietary assessment } \\
\hline & & Validated FFQ & 11 & 6286 & $1.30(1.02,1.66)$ & 85.6 & 0.000 \\
\hline & & FFQ & 5 & 823 & $1.68(0.67,4.20)$ & 87.3 & 0.000 \\
\hline & DLBCL & All studies & 3 & 352 & $1.49(1.08,2.06)$ & 8.9 & 0.333 \\
\hline & FL & All studies & 3 & 390 & $0.99(0.47,2.07)$ & 81.8 & 0.004 \\
\hline & SLL/CLL & All studies & 3 & 477 & $1.04(0.69,1.55)$ & 44.1 & 0.167 \\
\hline \multirow{14}{*}{ Cheese } & \multirow{11}{*}{ NHL } & All studies & 10 & 5519 & $1.14(0.96,1.34)$ & 58.2 & 0.011 \\
\hline & & \multicolumn{6}{|c|}{ Study design } \\
\hline & & Case-control & 9 & 4252 & $1.14(0.94,1.38)$ & 61.9 & 0.007 \\
\hline & & Cohort & 1 & 1267 & $1.09(0.85,1.39)$ & NA & NA \\
\hline & & \multicolumn{6}{|c|}{ Continent } \\
\hline & & North America & 5 & 2866 & $0.95(0.70,1.29)$ & 75.7 & 0.002 \\
\hline & & Europe & 4 & 2483 & $1.28(1.09,1.49)$ & 2.2 & 0.382 \\
\hline & & Asia & 1 & 170 & $1.38(0.79,2.41)$ & NA & NA \\
\hline & & \multicolumn{6}{|c|}{ Dietary assessment } \\
\hline & & Validated FFQ & 8 & 5204 & $1.24(1.09,1.40)$ & 23.1 & 0.245 \\
\hline & & FFQ & 2 & 315 & $0.64(0.44,0.91)$ & 0.0 & 0.686 \\
\hline & DLBCL & All studies & 3 & 352 & $0.93(0.63,1.37)$ & 0.0 & 0.905 \\
\hline & FL & All studies & 3 & 390 & $1.04(0.74,1.46)$ & 0.0 & 0.777 \\
\hline & SLL/CLL & All studies & 3 & 477 & $1.28(0.91,1.81)$ & 0.0 & 0.876 \\
\hline Butter & NHL & All studies & 4 & 1534 & $1.31(1.04,1.65)$ & 36.9 & 0.190 \\
\hline \multirow{4}{*}{ Yogurt } & NHL & All studies & 4 & 2372 & $0.78(0.54,1.12)$ & 81.6 & 0.001 \\
\hline & DLBCL & All studies & 3 & 352 & $0.90(0.67,1.21)$ & 0.0 & 0.402 \\
\hline & FL & All studies & 3 & 390 & $0.89(0.63,1.25)$ & 33.9 & 0.220 \\
\hline & SLL/CLL & All studies & 3 & 477 & $0.97(0.76,1.23)$ & 0.0 & 0.679 \\
\hline Ice cream & NHL & All studies & 4 & 1598 & $1.57(1.11,2.20)$ & 72.3 & 0.013 \\
\hline
\end{tabular}

Abbreviations: RR, relative risk; $\mathrm{CI}$, confidence interval; FFQ, food frequency questionnaire; NHL, non-Hodgkin lymphoma; DLBCL, diffuse large B-cell lymphoma; FL, follicular lymphoma; SLL/CLL, small lymphocytic lymphoma/chronic lymphocytic leukemia; NA, not available. 


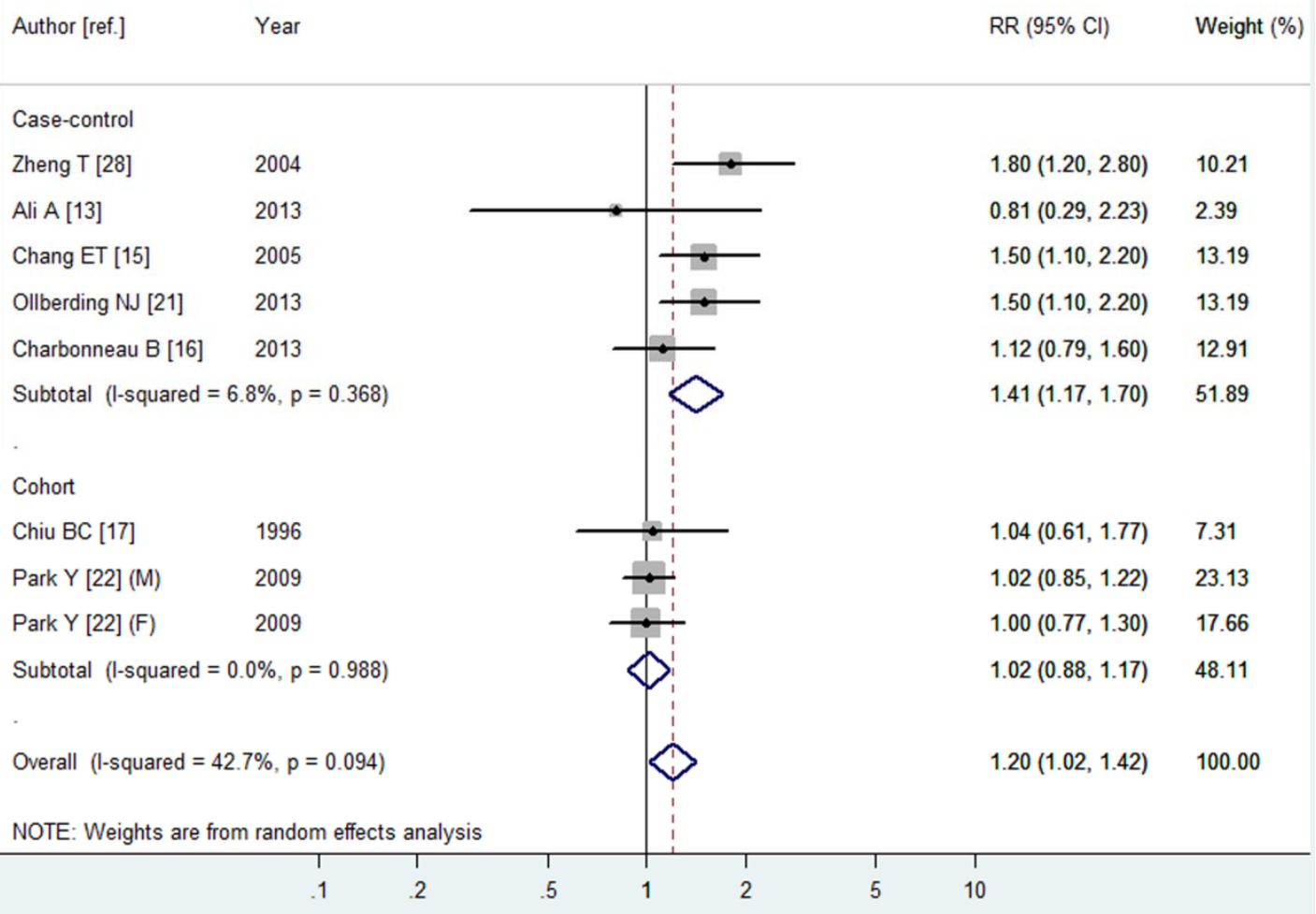

Figure 2. Forest plot of total dairy product consumption and the risk of NHL. The size of gray box is positively proportional to the weight assigned to each study, and horizontal lines represent the $95 \%$ confidence intervals.

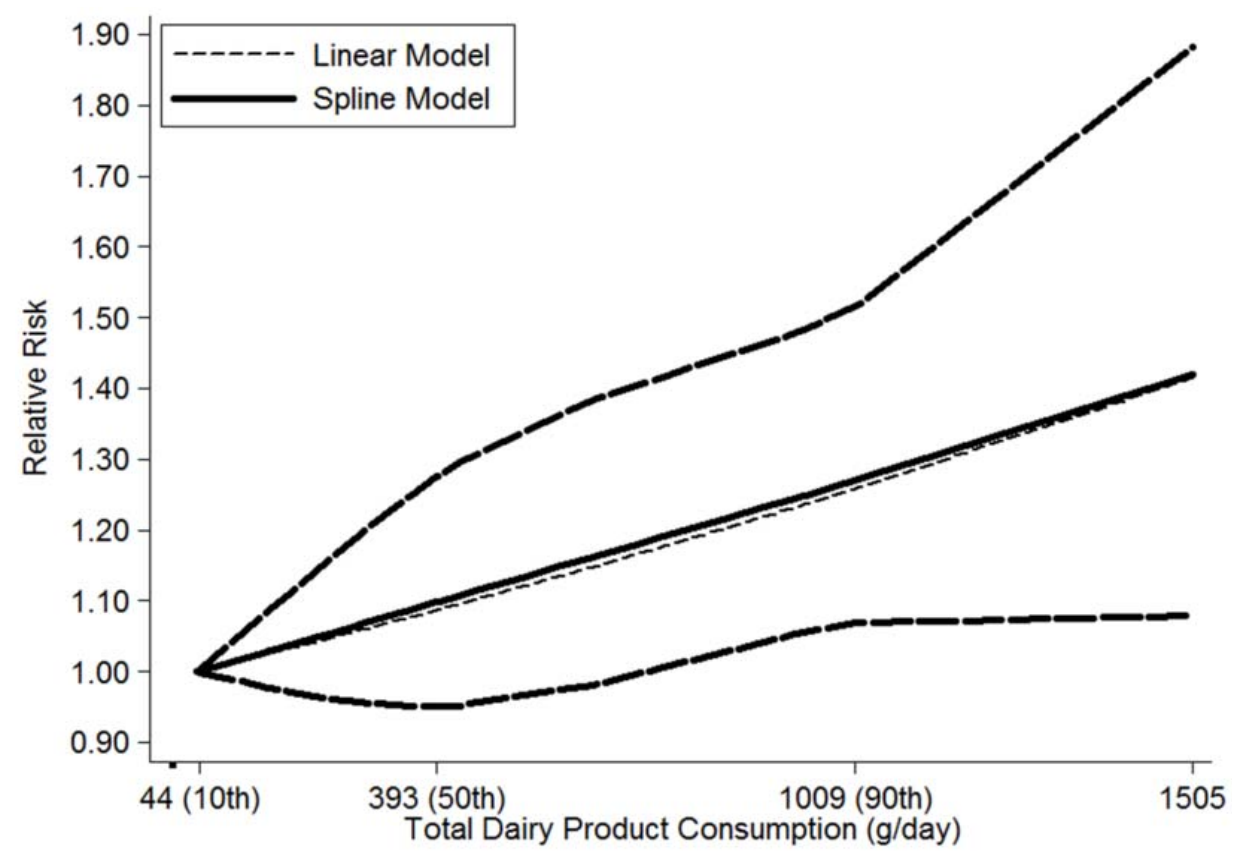

Figure 3. The dose-response analysis between total dairy product consumption and the risk of NHL with restricted cubic splines in a multivariate random-effects dose-response model. The solid line and the long dash line represent the estimated relative risks and their 95\% CIs. Short dash line represents the linear relationship. The 10th, 50th and 90th percentiles represent three knots of total dairy product consumption. 


\subsubsection{Milk Consumption and the Risk of NHL}

Fourteen articles [14-21,23-28] with 16 studies (14 case-control studies and two cohort studies) were included, involving 7109 NHL cases. Among these studies, seven were conducted in North America, three in Latin America, four in Europe and two in Asia. Eleven studies adopted validated FFQs to assess milk consumption and five studies adopted FFQs. For the highest vs. lowest category of milk consumption, the pooled RR of NHL was 1.41 (95\% CI $1.08-1.84, I^{2}=88.6 \%$, $P_{\text {heterogeneity }}=0.000$, Figure 4$)$. In subgroup analysis stratified by study design, the pooled RRs in case-control and cohort studies were $1.53\left(95 \%\right.$ CI 1.13-2.06, $I^{2}=87.7 \%, P$ heterogeneity $\left.=0.000\right)$ and $0.91\left(95 \%\right.$ CI $0.68-1.22, I^{2}=36.8 \%, P$ heterogeneity $\left.=0.209\right)$, respectively (Figure 4$)$. In subgroup analysis stratified by continent in which the studies were conducted, the positive association was statistically significant only among studies conducted in North America (RR $=1.21,95 \%$ CI 1.01-1.46, $I^{2}=37.8 \%, P$ heterogeneity $=0.140$ ). In subgroup analysis stratified by dietary assessment method, the positive association was statistically significant in studies that adopted validated FFQs $(R R=1.30$, $95 \%$ CI 1.02-1.66, $I^{2}=85.6 \%, P$ heterogeneity $\left.=0.000\right)$, but not in studies that used FFQs that had not been validated. In the further analysis of NHL subtypes, we just found a statistically significant association between milk consumption and the risk of DLBCL ( RR $=1.49,95 \%$ CI $1.08-2.06, I^{2}=8.9 \%$, $P$ heterogeneity $=0.333)$.

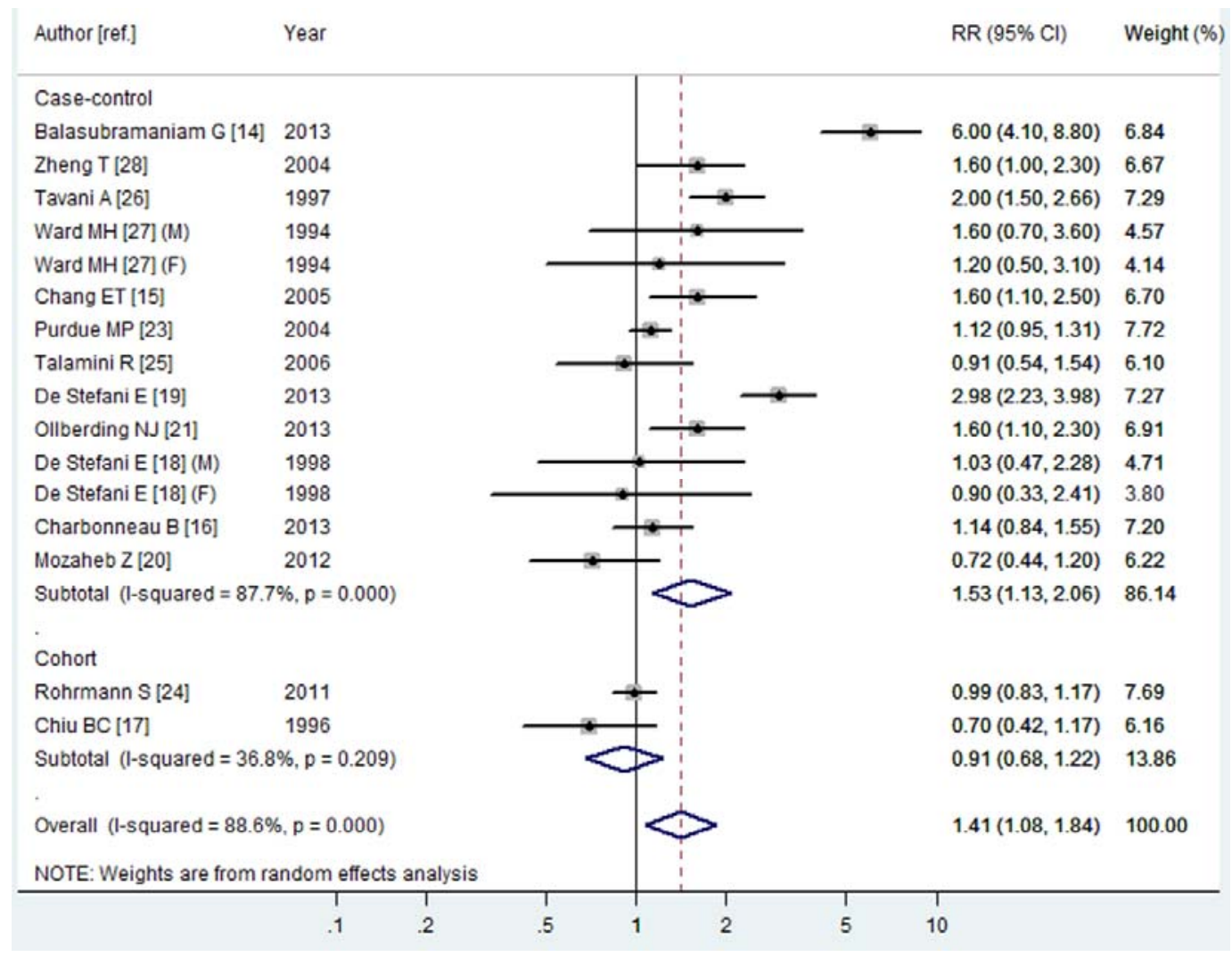

Figure 4. Forest plot of milk consumption and the risk of NHL. The size of the gray box is positively proportional to the weight assigned to each study, and horizontal lines represent the $95 \%$ confidence intervals.

For the dose-response analysis, data from nine studies [16-18,21,23,27,28] were included, including 3739 NHL cases. A linear relationship was found between milk consumption and the risk of NHL ( $P$ nonlinearity $=0.78)$, and the RRs (95\% CIs) of NHL were 1.04 (0.97-1.12), $1.07(0.96-1.19)$, 1.11 (0.99-1.24), $1.12(1.00-1.26)$ and 1.13 (1.00-1.28) for 120, 210, 370, 440 and $490 \mathrm{~g} /$ day compared 
with $0 \mathrm{~g} /$ day, respectively. In addition, the dose-response analysis suggested that NHL risk increased by $6 \%(1.06(0.99-1.13))$ for each $200 \mathrm{~g} /$ day increment of milk consumption (Figure 5).

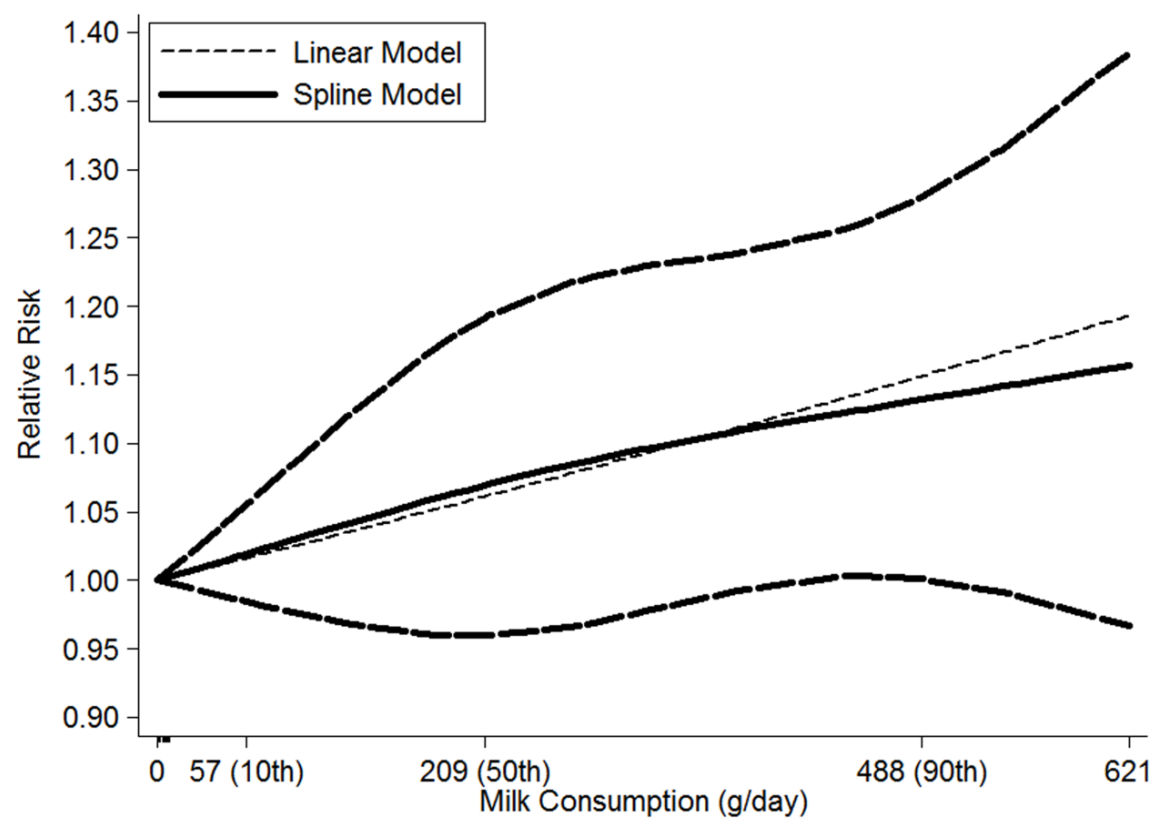

Figure 5. The dose-response analysis between milk consumption and the risk of NHL with restricted cubic splines in a multivariate random-effects dose-response model. The solid line and the long dash line represent the estimated relative risks and its 95\% CIs. Short dash line represents the linear relationship. The 10th, 50th and 90th percentiles represent three knots of milk consumption.

\subsubsection{Cheese Consumption and the Risk of NHL}

Nine articles $[15,16,20,21,23-27]$ with 10 studies (nine case-control studies and one cohort study) were included, involving 5519 NHL cases. Among these studies, five were conducted in North America, four in Europe and one in Asia. Eight studies adopted validated FFQs to assess cheese consumption and two studies adopted FFQs. The pooled RR of NHL was 1.14 (95\% CI 0.96-1.34, $I^{2}=58.2 \%, P$ heterogeneity $\left.=0.011\right)$ for the highest $v$ s. lowest category of consumption. In subgroup analysis stratified by study design, no association was found in both case-control studies and cohort studies. In subgroup analysis stratified by continent in which the studies were conducted, the positive association was statistically significant only among studies conducted in Europe ( $R R=1.28$, $95 \%$ CI 1.09-1.49, $I^{2}=2.2 \%, P_{\text {heterogeneity }}=0.382$ ). In subgroup analysis stratified by dietary assessment method, cheese consumption was associated with an increased risk of NHL (RR $=1.24,95 \%$ CI 1.09-1.40, $I^{2}=23.1 \%, P$ heterogeneity $\left.=0.245\right)$ in studies that adopted validated FFQs. In the further analysis of NHL subtypes, we did not find a statistically significant association between cheese consumption and any NHL subtypes.

\subsubsection{Other Dairy Product Consumption and the Risk of NHL}

For butter consumption, four articles $[16,20,21,26]$ with four case-control studies involving 1534 NHL cases were included, and the pooled RR of NHL was 1.31 (95\% CI 1.04-1.65, $I^{2}=36.9 \%$, $\left.p_{\text {heterogeneity }}=0.190\right)$ for the highest $v s$. lowest category of consumption. For yogurt consumption, four articles $[16,20,21,24]$ with four studies (three case-control studies and one cohort study) involving 2372 NHL cases were included, and the pooled RR of NHL was 0.78 (95\% CI $0.54-1.12, I^{2}=81.6 \%$, $\left.p_{\text {heterogeneity }}=0.001\right)$ for the highest $v s$. lowest category of consumption. In the further analysis of NHL subtypes, we did not find a statistically significant association between yogurt consumption and any NHL subtypes. For ice cream consumption, four articles $[16,20,21,28]$ with four case-control studies 
involving 1598 NHL cases were included, and the pooled RR of NHL was 1.57 (95\% CI 1.11-2.20, $I^{2}=72.3 \%, P$ heterogeneity $=0.013$ ) for the highest $v$ s. lowest category of consumption.

\subsection{Meta-Regression and Influence Analysis}

In order to explore the between-study heterogeneity, we performed univariate meta-regression with the covariates of sex, publication year, continent in which the studies were conducted, study design, dietary assessment method and whether the RR (95\% CI) was adjusted for energy intake, smoking, alcohol intake and education. In the analysis of total dairy product consumption and the risk of NHL, study design was found to contribute to the between-study heterogeneity $(p=0.011)$. In the analysis of cheese consumption and the risk of NHL, the dietary assessment method was found to contribute to the between-study heterogeneity $(p=0.018)$. None of these covariates was found to have a significant impact on the between-study heterogeneity in other analyses.

In an influence analysis excluding one study at a time, no individual study had an excessive influence on the above-mentioned pooled effects.

\subsection{Small-Study Effect Evaluation}

Egger's test showed no evidence of a significant small-study effect for the analyses between the consumption of total dairy product $(p=0.402)$, milk $(p=0.616)$, butter $(p=0.798)$, cheese $(p=0.278)$, yogurt $(p=0.196)$ and ice cream $(p=0.250)$ and the risk of NHL. The funnel plot of the analysis of milk consumption and the risk of NHL was shown in the Figure 6.

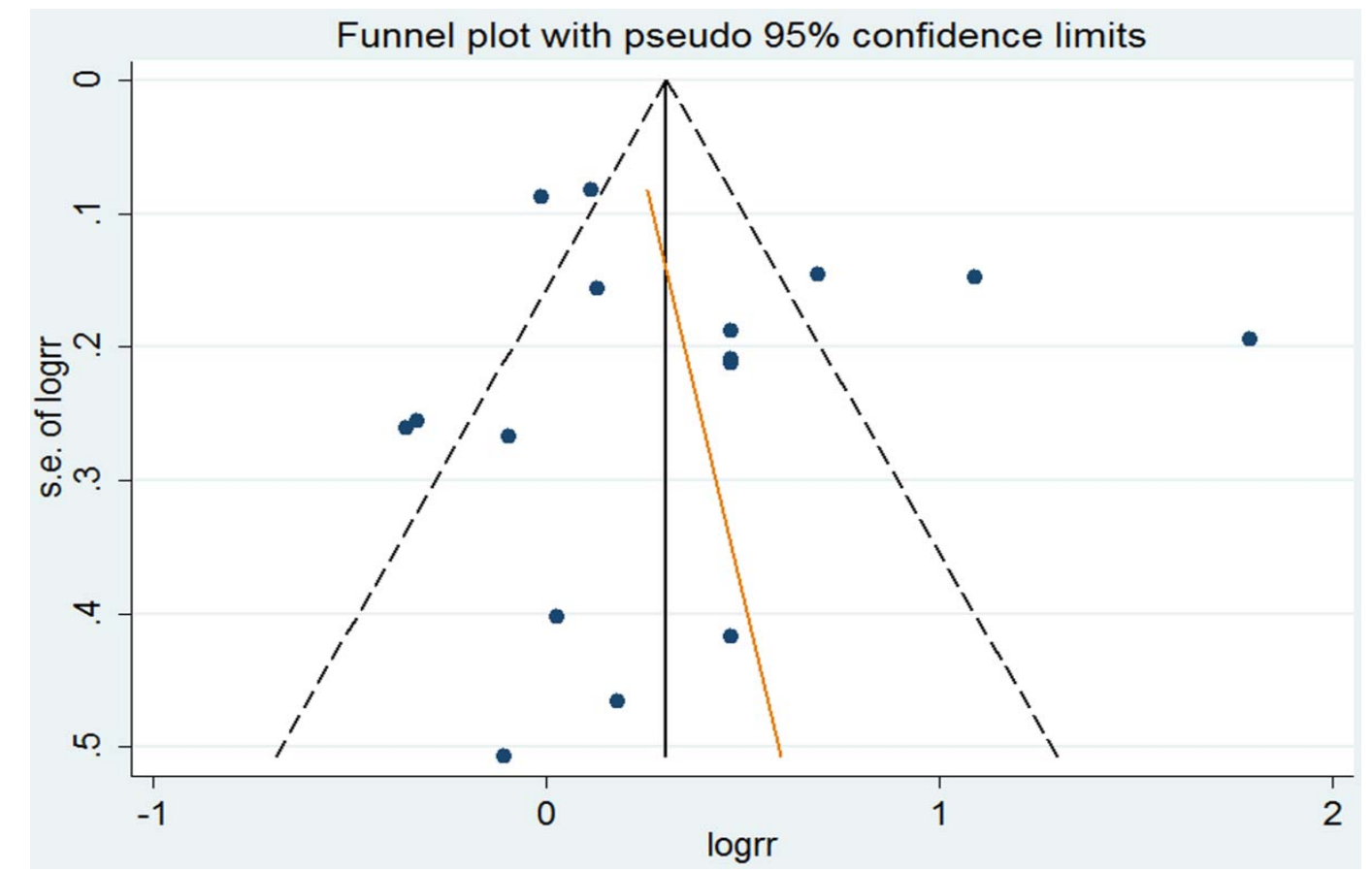

Figure 6. The funnel plot of milk consumption and the risk of NHL. Each dot represents a different study.

\section{Discussion}

This meta-analysis assessed associations between the consumption of total dairy product and specific types of dairy product and the risk of NHL, respectively. Findings from this meta-analysis showed positive associations between the consumption of total dairy product, milk, butter and ice cream and the risk of NHL, respectively. In subgroup analyses that were stratified by study design, only in case-control studies was the consumption of total dairy product and milk associated with 
an increased risk of NHL. In subgroup analyses that were stratified by the continent in which the studies were conducted, milk consumption was associated with an increased risk of NHL among studies conducted in North America, and cheese consumption was associated with an increased risk of NHL among studies conducted in Europe. When studies were stratified by the dietary assessment method, the consumption of milk and cheese were associated with an increased risk of NHL in those studies that used validated FFQs but not for other dietary assessment methods. Dose-response analysis suggested NHL risk increased by 5\% (1.05 (1.00-1.10)) and 6\% (1.06 (0.99-1.13)) for each $200 \mathrm{~g} / \mathrm{day}$ increment of total dairy product and milk consumption, respectively.

The mechanisms underlying the association between dairy product consumption and the risk of NHL have been postulated from several aspects. Dairy product is rich in protein and fat. Some experimental evidence from animal studies suggested that excessive intake of protein could give rise to chronic hyperstimulation of the immune system [39]. Several animal studies have shown that the changes of animal fat and protein in diet can cause impaired immune function [40], which is one of the few well-established risk factors for NHL [41]. An animal study with rats found an increased risk of lymphomas after augmentation of the diet with casein, the major protein of milk [42]. In addition, some epidemiologic studies $[43,44]$ suggested that polychlorinated dibenzo-p-dioxins (PCDDs) and polychlorinated dibenzofurans (PCDFs) could increase the risk of NHL. Several studies indicated that dairy was one of the most important contributors to total PCDDs and PCDFs intake in different regions [45-47]. Dairy product is also the main source of calcium. Several studies reported that high calcium intake could restrict the bioavailability of vitamin D $[48,49]$. An experimental study showed that 1,25-dihydroxyvitamin $\mathrm{D}_{3}$, the active form of vitamin $\mathrm{D}$, had an anti-proliferative and pro-differentiation effect on cells that possess vitamin D receptors in follicular lymphoma cell lines [50]. However, a meta-analysis indicated that higher vitamin $\mathrm{D}$ did not play a protective role in the risk of NHL [51]. So far, the effect of vitamin D on the association between dairy product consumption and the risk of NHL is still uncertain. More research is needed to confirm this effect. In this meta-analysis, an inverse but not significant association was found between yogurt consumption and the risk of NHL. The anticancer effect of yogurt may depend on the large amounts of lactic acid bacteria, which can enhance the immune response of the host and exert antioxidative and antiproliferative activity [52,53].

Between-study heterogeneity is common in meta-analysis [54]. It is necessary to explore the potential sources of between-study heterogeneity. Our meta-analysis showed different levels of between-study heterogeneities in the analyses of total dairy product and specific types of dairy product consumption and risk of NHL. In meta-regression, we found that study design and the dietary assessment method were the contributors to between-study heterogeneities in the analyses of total dairy product and cheese consumption, respectively. Case-control studies are more susceptible to recall and selection biases than cohort studies. Recall bias is the most common and inevitable bias in all case-control studies, and it refers to the differential recollection of dairy product consumption between cases and controls. The most common selection bias is that the controls are from the hospital. These may contribute to the between-study heterogeneity. Subgroup analysis stratified by dietary assessment method showed that cheese consumption can increase the risk of NHL in studies that adopted validated FFQs. The validated FFQs can provide more accurate dietary intake and make the results more credible. However, meta-regression did not find the sources of between-study heterogeneities in other analyses. The factors accounting for the heterogeneity between studies are complicated. First, the methodologies among the included studies were different, such as the dietary assessment method. Second, the consumption levels ranged widely across the studies included in this meta-analysis. For total dairy product consumption, the lowest intake categories ranged from $<3$ servings / week to 2.4 servings/day, and the highest intake categories ranged from $>1.2$ servings/day to 8.5 servings/day. For milk consumption, the lowest intake categories ranged from 0 to $<6.9$ servings/week, and the highest intake categories ranged from $>4$ servings / week to $>14$ servings / week. Third, NHL refers to a heterogeneous group of lymphomas with different prognoses and possible different etiology. The proportion of different NHL subtypes in the included studies may be different. Fourth, the 
consumption amount and type of dairy product largely differed between Western countries and Eastern countries. All of these factors may contribute to the between-study heterogeneity in concert.

To our knowledge, this is the first meta-analysis to explore the associations between the consumption of total dairy product and specific types of dairy product and the risk of NHL. A major strength of this meta-analysis is the large number of cases included, increasing the statistical power of the study to detect the associations. Second, RRs that reflected the greatest degree of control for potential confounders were extracted, indicating that the results were more credible. Third, considering the potential differences of components in different types of dairy product, we further assessed the effects of specific types of dairy product on the risk of NHL. Fourth, dose-response analysis was conducted to explore the relationships between the consumption of total dairy product and milk and the risk of NHL quantitatively. Fifth, almost all the included studies used a validated FFQ, which ensured the credibility of dietary assessment.

However, there are some limitations in this meta-analysis. First, our results mainly came from case-control studies. There were only three cohort studies included in this meta-analysis, and the positive association between total dairy product consumption and the risk of NHL was not statistically significant in cohort studies. In addition, two other cohort studies $[55,56]$ without $\mathrm{RR}$ and/or $95 \% \mathrm{CI}$ were not included in our meta-analysis. One [55] of them indicated that the consumption of more than two glasses of milk per day could increase the risk of NHL, but another [56] found no statistically significant association between the consumption of any type of dairy product and the risk of NHL. Therefore, more cohort studies with complete data are needed to confirm these results. Second, although major confounders had been adjusted for in most of the included studies, unmeasured and residual confounding was still possible. Confounders adjusted for in each study were also different, which might affect the observed association. Third, the numbers of studies on the consumption of butter, yogurt and ice cream were limited. Thus, fewer cases reduced the statistical power to detect a statistically significant association. Fourth, because of the limited number of studies, we could not evaluate the association between the consumption of dairy product with different fat content and the risk of NHL. Fifth, because of the limited number of studies which examined the associations with specific histopathological subtypes of NHL, we only explored the associations between dairy product consumption and the risk of DLBCL, follicular lymphoma (FL), and small lymphocytic lymphoma/chronic lymphocytic leukemia (SLL/CLL). The fewer studies and cases reduced the statistical power to detect statistically significant associations. Sixth, in dose-response analysis, we assumed that the amplitude of the highest category is same as the contiguous category. This may be imprecise given the asymmetric distributions of food consumption. Considering that most of the studies in dose-response analysis were conducted in the US, we adopted standard units reported in the United States Department of Agriculture National Nutrient Database for Standard Reference to convert our data for all the included studies. These may affect the observed association to some extent.

\section{Conclusions}

In summary, this meta-analysis suggested that dairy product, but not yogurt, may increase the risk of NHL. The risk of NHL increased by $5 \%$ and $6 \%$ for each $200 \mathrm{~g}$ /day increment of total dairy product and milk consumption, respectively. The results mainly came from case-control studies, and thus more cohort studies focusing on specific types of dairy product consumption are needed to confirm the conclusion.

Acknowledgments: The conduct of this study was not funded.

Author Contributions: Jia Wang and Dongfeng Zhang designed the study. Jia Wang and Xutong Li carried out the literature search and data extraction. Jia Wang and Xutong Li carried out the statistical analysis. Jia Wang and Dongfeng Zhang were involved in drafting and revising the manuscript. All authors reviewed and approved the manuscript.

Conflicts of Interest: The authors declare no conflict of interest. 


\section{References}

1. Huncharek, M.; Muscat, J.; Kupelnick, B. Impact of dairy products and dietary calcium on bone-mineral content in children: Results of a meta-analysis. Bone 2008, 43, 312-321. [CrossRef] [PubMed]

2. Qin, L.Q.; Xu, J.Y.; Han, S.F.; Zhang, Z.L.; Zhao, Y.Y.; Szeto, I.M. Dairy consumption and risk of cardiovascular disease: An updated meta-analysis of prospective cohort studies. Asia Pac. J. Clin. Nutr. 2015, 24, 90-100. [PubMed]

3. Chen, M.; Sun, Q.; Giovannucci, E.; Mozaffarian, D.; Manson, J.E.; Willett, W.C.; Hu, F.B. Dairy consumption and risk of type 2 diabetes: 3 cohorts of US adults and an updated meta-analysis. BMC Med. 2014, 12, 215. [CrossRef] [PubMed]

4. Cho, E.; Smith-Warner, S.A.; Spiegelman, D.; Beeson, W.L.; van den Brandt, P.A.; Colditz, G.A.; Folsom, A.R.; Fraser, G.E.; Freudenheim, J.L.; Giovannucci, E.; et al. Dairy foods, calcium, and colorectal cancer: A pooled analysis of 10 cohort studies. J. Natl. Cancer Inst. 2004, 96, 1015-1022. [CrossRef] [PubMed]

5. Jiang, W.; Ju, C.; Jiang, H.; Zhang, D. Dairy foods intake and risk of Parkinson's disease: A dose-response meta-analysis of prospective cohort studies. Eur. J. Epidemiol 2014, 29, 613-619. [CrossRef] [PubMed]

6. Aune, D.; Navarro Rosenblatt, D.A.; Chan, D.S.; Vieira, A.R.; Vieira, R.; Greenwood, D.C.; Vatten, L.J.; Norat, T. Dairy products, calcium, and prostate cancer risk: A systematic review and meta-analysis of cohort studies. Am. J. Clin. Nutr. 2015, 101, 87-117. [CrossRef] [PubMed]

7. Cartwright, R.; Brincker, H.; Carli, P.M.; Clayden, D.; Coebergh, J.W.; Jack, A.; McNally, R.; Morgan, G.; de Sanjose, S.; Tumino, R.; et al. The rise in incidence of lymphomas in Europe 1985-1992. Eur. J. Cancer 1999, 35, 627-633. [CrossRef]

8. Devesa, S.S.; Fears, T. Non-Hodgkin's lymphoma time trends: United States and international data. Cancer Res. 1992, 52, 5432s-5440s. [PubMed]

9. Levi, F.; Lucchini, F.; Negri, E.; La Vecchia, C. Trends in mortality from non-Hodgkin's lymphomas. Leuk. Res. 2002, 26, 903-908. [CrossRef]

10. Siegel, R.L.; Miller, K.D.; Jemal, A. Cancer statistics, 2015. CA Cancer J. Clin. 2015, 65, 5-29. [CrossRef] [PubMed]

11. Chen, G.C.; Lv, D.B.; Pang, Z.; Liu, Q.F. Fruits and vegetables consumption and risk of non-Hodgkin's lymphoma: A meta-analysis of observational studies. Int. J. Cancer 2013, 133, 190-200. [CrossRef] [PubMed]

12. Fallahzadeh, H.; Cheraghi, M.; Amoori, N.; Alaf, M. Red meat intake and risk of non-Hodgkin lymphoma: A meta-analysis. Asian Pac. J. Cancer Prev. 2014, 15, 10421-10425. [CrossRef] [PubMed]

13. Ali, A.; Al-Belushi, B.S.; Waly, M.I.; Al-Moundhri, M.; Burney, I.A. Dietary and lifestyle factors and risk of non-Hodgkin's lymphoma in Oman. Asian Pac. J. Cancer Prev. 2013, 14, 841-848. [CrossRef] [PubMed]

14. Balasubramaniam, G.; Saoba, S.; Sarade, M.; Pinjare, S. Case-control study of risk factors for non-Hodgkin lymphoma in Mumbai, India. Asian Pac. J. Cancer Prev. 2013, 14, 775-780. [CrossRef] [PubMed]

15. Chang, E.T.; Smedby, K.E.; Zhang, S.M.; Hjalgrim, H.; Melbye, M.; Ost, A.; Glimelius, B.; Wolk, A.; Adami, H.O. Dietary factors and risk of non-Hodgkin lymphoma in men and women. Cancer Epidemiol. Biomark. Prev. 2005, 14, 512-520. [CrossRef] [PubMed]

16. Charbonneau, B.; O'Connor, H.M.; Wang, A.H.; Liebow, M.; Thompson, C.A.; Fredericksen, Z.S.; Macon, W.R.; Slager, S.L.; Call, T.G.; Habermann, T.M.; et al. Trans fatty acid intake is associated with increased risk and $\mathrm{n} 3$ fatty acid intake with reduced risk of non-Hodgkin lymphoma. J. Nutr. 2013, 143, 672-681. [CrossRef] [PubMed]

17. Chiu, B.C.; Cerhan, J.R.; Folsom, A.R.; Sellers, T.A.; Kushi, L.H.; Wallace, R.B.; Zheng, W.; Potter, J.D. Diet and risk of non-Hodgkin lymphoma in older women. JAMA 1996, 275, 1315-1321. [CrossRef] [PubMed]

18. De Stefani, E.; Fierro, L.; Barrios, E.; Ronco, A. Tobacco, alcohol, diet and risk of non-Hodgkin's lymphoma: A case-control study in Uruguay. Leuk. Res. 1998, 22, 445-452. [CrossRef]

19. De Stefani, E.; Ronco, A.L.; Deneo-Pellegrini, H.; Boffetta, P.; Correa, P.; Barrios, E.; Acosta, G.; Mendilaharsu, M. Meat, milk and risk of lymphoid malignancies: A case-control study in Uruguay. Nutr. Cancer 2013, 65, 375-383. [CrossRef] [PubMed]

20. Mozaheb, Z.; Aledavood, A.; Farzad, F. Diet and non-Hodgkin's lymphoma risk. Pan. Afr. Med. J. 2012, 12, 53. [PubMed]

21. Ollberding, N.J.; Aschebrook-Kilfoy, B.; Caces, D.B.; Wright, M.E.; Weisenburger, D.D.; Smith, S.M.; Chiu, B.C. Phytanic acid and the risk of non-Hodgkin lymphoma. Carcinogenesis 2013, 34, 170-175. [CrossRef] [PubMed] 
22. Park, Y.; Leitzmann, M.F.; Subar, A.F.; Hollenbeck, A.; Schatzkin, A. Dairy food, calcium, and risk of cancer in the NIH-AARP Diet and Health Study. Arch. Intern. Med. 2009, 169, 391-401. [CrossRef] [PubMed]

23. Purdue, M.P.; Bassani, D.G.; Klar, N.S.; Sloan, M.; Kreiger, N.; Canadian Cancer Registries Epidemiology Research, G. Dietary factors and risk of non-Hodgkin lymphoma by histologic subtype: A case-control analysis. Cancer Epidemiol. Biomark. Prev. 2004, 13, 1665-1676.

24. Rohrmann, S.; Linseisen, J.; Jakobsen, M.U.; Overvad, K.; Raaschou-Nielsen, O.; Tjonneland, A.; Boutron-Ruault, M.C.; Kaaks, R.; Becker, N.; Bergmann, M.; et al. Consumption of meat and dairy and lymphoma risk in the European Prospective Investigation into Cancer and Nutrition. Int. J. Cancer 2011, 128, 623-634. [CrossRef] [PubMed]

25. Talamini, R.; Polesel, J.; Montella, M.; Dal Maso, L.; Crovatto, M.; Crispo, A.; Spina, M.; Canzonieri, V.; La Vecchia, C.; Franceschi, S. Food groups and risk of non-Hodgkin lymphoma: A multicenter, case-control study in Italy. Int. J. Cancer 2006, 118, 2871-2876. [CrossRef] [PubMed]

26. Tavani, A.; Pregnolato, A.; Negri, E.; Franceschi, S.; Serraino, D.; Carbone, A.; La Vecchia, C. Diet and risk of lymphoid neoplasms and soft tissue sarcomas. Nutr. Cancer 1997, 27, 256-260. [CrossRef] [PubMed]

27. Ward, M.H.; Zahm, S.H.; Weisenburger, D.D.; Gridley, G.; Cantor, K.P.; Saal, R.C.; Blair, A. Dietary factors and non-Hodgkin's lymphoma in Nebraska (United States). Cancer Causes Control 1994, 5, 422-432. [CrossRef] [PubMed]

28. Zheng, T.; Holford, T.R.; Leaderer, B.; Zhang, Y.; Zahm, S.H.; Flynn, S.; Tallini, G.; Zhang, B.; Zhou, K.; Owens, P.H.; et al. Diet and nutrient intakes and risk of non-Hodgkin's lymphoma in Connecticut women. Am. J. Epidemiol. 2004, 159, 454-466. [CrossRef] [PubMed]

29. US Department of Agriculture, Agricultural Research Service. USDA National Nutrient Database for Standard Reference, Release 28. Available online: http://ndb.nal.usda.gov/ndb/search. (accessed on 10 November 2015).

30. DerSimonian, R.; Laird, N. Meta-analysis in clinical trials. Control Clin. Trials 1986, 7, 177-188. [CrossRef]

31. Higgins, J.P.; Thompson, S.G.; Deeks, J.J.; Altman, D.G. Measuring inconsistency in meta-analyses. BMJ 2003, 327, 557-560. [CrossRef] [PubMed]

32. Higgins, J.P.; Thompson, S.G. Controlling the risk of spurious findings from meta-regression. Stat. Med. 2004, 23, 1663-1682. [CrossRef] [PubMed]

33. Tobias, A. Assessing the influence of a single study in the meta-analysis estimate. Stata Tech. Bull. 1999, 47, 15-17.

34. Egger, M.; Davey Smith, G.; Schneider, M.; Minder, C. Bias in meta-analysis detected by a simple, graphical test. BMJ 1997, 315, 629-634. [CrossRef] [PubMed]

35. Orsini, N.; Li, R.; Wolk, A.; Khudyakov, P.; Spiegelman, D. Meta-analysis for linear and nonlinear dose-response relations: Examples, an evaluation of approximations, and software. Am. J. Epidemiol. 2012, 175, 66-73. [CrossRef] [PubMed]

36. Harrell, F.E., Jr.; Lee, K.L.; Pollock, B.G. Regression models in clinical studies: Determining relationships between predictors and response. J. Natl. Cancer Inst. 1988, 80, 1198-1202. [CrossRef] [PubMed]

37. Orsini, N.; Bellocco, R. Generalized least squares for trend estimation of summarized dose-response data. Stata J. 2006, 6, 40-57.

38. Jackson, D.; White, I.R.; Thompson, S.G. Extending DerSimonian and Laird's methodology to perform multivariate random effects meta-analyses. Stat. Med. 2010, 29, 1282-1297. [CrossRef] [PubMed]

39. Cunningham, A.S. Lymphomas and animal-protein consumption. Lancet 1976, 2, 1184-1186. [CrossRef]

40. Davis, S. Nutritional factors and the development of non-Hodgkin's lymphoma: A review of the evidence. Cancer Res. 1992, 52, 5492s-5495s. [PubMed]

41. Kinlen, L.J.; Sheil, A.G.; Peto, J.; Doll, R. Collaborative United Kingdom-Australasian study of cancer in patients treated with immunosuppressive drugs. Br. Med. J. 1979, 2, 1461-1466. [CrossRef] [PubMed]

42. Ross, M.H.; Bras, G. Tumor incidence patterns and nutrition in the rat. J. Nutr. 1965, 87, 245-260. [PubMed]

43. Engel, L.S.; Laden, F.; Andersen, A.; Strickland, P.T.; Blair, A.; Needham, L.L.; Barr, D.B.; Wolff, M.S.; Helzlsouer, K.; Hunter, D.J.; et al. Polychlorinated biphenyl levels in peripheral blood and non-Hodgkin's lymphoma: A report from three cohorts. Cancer Res. 2007, 67, 5545-5552. [CrossRef] [PubMed]

44. Spinelli, J.J.; Ng, C.H.; Weber, J.P.; Connors, J.M.; Gascoyne, R.D.; Lai, A.S.; Brooks-Wilson, A.R.; Le, N.D.; Berry, B.R.; Gallagher, R.P. Organochlorines and risk of non-Hodgkin lymphoma. Int. J. Cancer 2007, 121, 2767-2775. [CrossRef] [PubMed] 
45. Baars, A.J.; Bakker, M.I.; Baumann, R.A.; Boon, P.E.; Freijer, J.I.; Hoogenboom, L.A.; Hoogerbrugge, R.; van Klaveren, J.D.; Liem, A.K.; Traag, W.A.; et al. Dioxins, dioxin-like PCBs and non-dioxin-like PCBs in foodstuffs: Occurrence and dietary intake in the netherlands. Toxicol. Lett. 2004, 151, 51-61. [CrossRef] [PubMed]

46. Bocio, A.; Domingo, J.L. Daily intake of polychlorinated dibenzo-p-dioxins/polychlorinated dibenzofurans (PCDD/PCDFs) in foodstuffs consumed in Tarragona, Spain: A review of recent studies (2001-2003) on human PCDD/PCDF exposure through the diet. Environ. Res. 2005, 97, 1-9. [CrossRef] [PubMed]

47. Fattore, E.; Fanelli, R.; Turrini, A.; di Domenico, A. Current dietary exposure to polychlorodibenzo-p-dioxins, polychlorodibenzofurans, and dioxin-like polychlorobiphenyls in Italy. Mol. Nutr. Food Res. 2006, 50, 915-921. [CrossRef] [PubMed]

48. Giovannucci, E. Dietary influences of $1,25(\mathrm{OH})_{2}$ vitamin D in relation to prostate cancer: A hypothesis. Cancer Causes Control 1998, 9, 567-582. [CrossRef] [PubMed]

49. Giovannucci, E.; Liu, Y.; Stampfer, M.J.; Willett, W.C. A prospective study of calcium intake and incident and fatal prostate cancer. Cancer Epidemiol. Biomark. Prev. 2006, 15, 203-210. [CrossRef] [PubMed]

50. Hickish, T.; Cunningham, D.; Colston, K.; Millar, B.C.; Sandle, J.; Mackay, A.G.; Soukop, M.; Sloane, J. The effect of 1,25-dihydroxyvitamin D3 on lymphoma cell lines and expression of vitamin D receptor in lymphoma. Br. J. Cancer 1993, 68, 668-672. [CrossRef] [PubMed]

51. Lu, D.; Chen, J.; Jin, J. Vitamin D status and risk of non-Hodgkin lymphoma: A meta-analysis. Cancer Causes Control 2014, 25, 1553-1563. [CrossRef] [PubMed]

52. Kim, J.E.; Kim, J.Y.; Lee, K.W.; Lee, H.J. Cancer chemopreventive effects of lactic acid bacteria. J. Microbiol. Biotechnol. 2007, 17, 1227-1235. [PubMed]

53. Perdigon, G.; de Moreno de LeBlanc, A.; Valdez, J.; Rachid, M. Role of yoghurt in the prevention of colon cancer. Eur. J. Clin. Nutr. 2002, 56 (Suppl. 3), S65-S68. [CrossRef] [PubMed]

54. Munafo, M.R.; Flint, J. Meta-analysis of genetic association studies. Trends Genet. 2004, 20, 439-444. [CrossRef] [PubMed]

55. Ursin, G.; Bjelke, E.; Heuch, I.; Vollset, S.E. Milk consumption and cancer incidence: A Norwegian prospective study. Br. J. Cancer 1990, 61, 454-459. [CrossRef] [PubMed]

56. Zhang, S.; Hunter, D.J.; Rosner, B.A.; Colditz, G.A.; Fuchs, C.S.; Speizer, F.E.; Willett, W.C. Dietary fat and protein in relation to risk of non-Hodgkin's lymphoma among women. J. Natl. Cancer Inst. 1999, 91, 1751-1758. [CrossRef] [PubMed] 